Sección II HABLANDO DE... 



\title{
Mercantilización de la Educación. Comentarios sobre la Reforma Educativa en Chile 2015.
}

\author{
Marketing of Education. Commentaries upon the \\ Educational Reform in Chile 2015
}

\author{
Juan González López \\ Psicólogo \\ Especialista en Políticas Públicas \\ Dpto. Educación. Universidad de Chile \\ Capitán Ignacio Carrera Pinto 1045 \\ juangl@uchile.cl \\ Diego Parra Moreno \\ Psicólogo \\ Especialista en Políticas Públicas \\ Centro ALERTA \\ diegoparam@gmail.com
}

Resumen: El presente texto pretende analizar las medidas contenidas en la llamada ley de inclusión educativa. Estas medidas han sido promovidas en la ciudadanía señalando que responden a las demandas del movimiento social por la educación que se ha movilizado en escuelas y calles, año a año desde el 2006. Se hará un análisis de estas medidas, contextualizando la reforma chilena en la reforma educativa neoliberal que se está implementando en el continente desde los años 80 y utilizando algunos datos sobre la privatización del sistema educativo chileno recogidos en proceso de investigación. ${ }^{1}$

Palabras claves: mediciones, inclusión educacional, reforma, privatización

Abstract: The purpose of this paper is to analyze the procedures contained in the so-called law of educational inclusion. These procedures are presented to the citizenry as an answer to the demands of the social movement for education, which has come forward in the schools and in the streets year after year since 2006. An analysis is made of these changes and adjustments by placing the Chilean reform within the context of the neoliberal educational reform of the 80 s and by availing ourselves of of some data on the privatization of the Chilean educational system, data which we gathered along our research.

Key Words: measures, educational inclusion, reform, privatization. 


\section{INTRODUCCIÓN}

Comentar la reforma chilena necesariamente lleva a reflexionar sobre el neoliberalismo y la privatización, en tanto estamos hablando de la transformación del sistema educativo más privatizado, en el país más neoliberal, de la región más neoliberal del mundo (Sader, 2006). Terminar con el lucro e implementar la gratuidad, parece ser el giro que necesita el modelo y lo que pide el movimiento social por la educación que, al menos desde el 2001 se ha movilizado (OPECH, 2009). Sin embargo ¿es posible dar este giro sólo con estas medidas?

Para analizar esto es necesario situar la reforma chilena actual en un relato que tome la historia reciente de reforma educativa en Latinoamérica y los resultados del proceso privatizador en Chile. Así se tendrán elementos que permitan hacer algunas reflexiones sobre el carácter y la proyección de esta reforma. Relevante discusión en tanto estas medidas son apreciadas en la región como un freno a las políticas neoliberales que vienen implementándose en Chile hace 35 años.

\section{LA LEY DE INCLUSIÓN Y EL DEBATE EDUCATIVO ACTUAL}

La llamada ley de inclusión educativa es un complejo de nuevos artículos, ajustes y eliminación de párrafos a distintas leyes que regulan el sistema educativo ${ }^{2}$. Estos cambios en su conjunto son lo que finalmente constituye la ley que regularía la admisión de los y las estudiantes, elimina el financiamiento compartido y prohíbe el lucro en establecimientos educacionales que reciben aportes del Estado. Una reforma de difícil análisis e interpretación para la ciudadanía, que se anunció el 19 de Mayo del 2014 y se aprobó en el congreso el 22 de Enero del 2015, en período vacaciones. Tendría que entrar, parcialmente, en funcionamiento en abril del 2016, casi 10 años después de iniciadas las movilizaciones de estudiantes secundarios que comenzaron a resistir el terremoto neoliberal, ${ }^{3}$ tras las consignas de fin al lucro y gratuidad en la educación.

Esta ley supone ser la reforma estructural ${ }^{4}$ al sistema educativo y uno de los ejes centrales del programa por el que fue electo el actual gobierno. En este sentido constituye el puntapié inicial de la eliminación de la excesiva influencia de los mecanismos de mercado y competencia en el sistema educativo chileno (OCDE, 2004, p. 290). Además, hasta la fecha, es la única modificación legal en educación aprobada en el parlamento en este gobierno reformista, que se ha comprometido a dar un giro en la lógica en que se ha venido construyendo nuestro sistema educativo. 


\section{CONTEXTO: LAS REFORMAS NEOLIBERALES EN LA REGIÓN.}

En Chile a partir de los 80 comienza la implementación de medidas para desarticular la educación estatal. Se entrega la gestión de escuelas a privados, se reduce el gasto en educación, y se modifica el marco normativo, respecto al derecho a la educación y al rol del Estado 5 . Bajo el argumento de la descentralización educativa, se congeló la inversión del Estado (incluso se redujo) ${ }^{6}$ y se liberalizó la oferta privada, basados en la libertad de enseñanza, como marco legal y la competencia como marco ideológico. Señala Gerardo Jofré, ingeniero comercial, arquitecto de estas primeras reformas en Chile. Un sistema basado en establecimientos particulares con fines de lucro, tiende no sólo a financiarse, sino a obtener el máximo aprovechamiento de los recursos: "El dolor pecuniario tiende a corregir vehementemente cualquier asomo de irresponsabilidad”. (Jofré, 1988, p. 209). También se precarizaron las condiciones de trabajo de los docentes. Desde el año 1974 a 1990 las remuneraciones docentes en Chile se estancaron con respecto al resto de las profesiones (Riesco, 2011, p. 3). La privatización del sistema avanzó significativamente en pocos años. Si en el año 1981, el 78\% de la matrícula se concentraba en escuelas estatales, al año 1990, sólo el 54\% de la matrícula estaba en escuelas del Estado (Assael y otros, 2011).

\section{Estas reformas educativas neoliberales de primera y segunda generación} están orientadas a la apertura del sistema educativo al mercado y la transformación radical de la escuela pública.

Principalmente impulsadas por el Banco Mundial, tuvieron su piloto en Chile, pero han seguido por países como Brasil, Perú, Argentina y Uruguay (Puiggrós, 2014 , p. 1). Ya en los años '90 este proceso se extiende a gran parte de la región, y comienza una nueva generación de medidas y discursos concomitantes con la privatización de las escuelas y con la nueva gestión del Estado. La modernización de los sistemas educativos, introduce la estandarización y toda una serie de ajustes, que tras la retórica de la eficacia y la calidad, orientan la escuela únicamente a resultados medibles y estándar, "una nueva forma de hacer escuela” se cierne sobre América Latina (Mejías, 2008, p. 5).7

Señala Marco Raúl Mejía: “...el capitalismo globalizado refunda su escuela: 122 nuevas leyes de educación en el mundo y 25 en América Latina. Asistimos en 22 años (1986-2008) a cuatro generaciones de reformas educativas. La primera, la de descentralización neoliberal, que tuvo su lugar experimental en las dictaduras de Corea, Chile, Argentina; la segunda, o de leyes generales de educación, tiene que ver con la modificación de las leyes nacionales de educación 
para colocarlas a tono con el capitalismo globalizado (en Colombia, Ley 115 de 1994); y la tercera, o de contrarreformas educativas, colocaron la educación como un gasto y la reestructuración se realiza en términos de eficiencia y eficacia según la racionalización neoliberal de la producción fabril y son hijas del ajuste fiscal neoliberal (en Colombia, ley 715 de 2002). En los últimos 15 años, la mayoría de ellas se han hecho con el patrocinio y asesoría del Banco Mundial". (Mejía, 2008, p. 6). También en Argentina se hace un radical recorte en el gasto que llevó a los docentes a protestar durante tres años (1997-1999) fuera del congreso nacional por una nueva Ley de Educación. En Brasil se imponen medidas de estandarización y homogeneización del sistema educativo, como el Sistema Nacional de Evaluación de la Enseñanza Básica (SAEB), el Examen Nacional de Enseñanza Media (ENEM), el Examen Nacional de Cursos (pruebón) (CLACSO, 2005, p. 227), entre otras. En 1992, se promueve en Colombia el Plan de Apertura Educativa, que implica la descentralización del sistema educativo. En Uruguay se acentúan los procesos de desprofesionalización y precarización docente (CLACSO, 2005). A esto sumamos la marcada incidencia mediante financiamiento, proyectos, programas, propuestas, de los organismos financieros internacionales en la política educativa de la región (Guerra, 2006). El proceso privatizador es complejo e implica una variedad de procesos concomitantes, que disuelven la escuela estatal y constituyen un nuevo modelo.

Es la subsunción real ${ }^{8}$ de la escuela al mercado. Proceso difícil de distinguir debido a su heterogéneo desarrollo en los distintos ámbitos de los sistemas educativos, imaginarios sociales y relaciones de poder. Pese a eso se distinguen al menos, tres dimensiones que se superponen y se relacionan entre sí. La exoprivatización, es decir la delegación de la gestión educativa a privados, tanto, en escuelas, como en servicios asociados a la educación pública, la llamada Nueva Gestión Pública9 . La endoprivatización, entendida como la transferencia de la gestión de la empresa privada a la escuela pública, la transformación de la misma en base a la lógica de la empresa actual ${ }^{10}$. Finalmente, la síntesis, y nuevo orden educativo, el control corporativo de la educación. Entendido este como el resultado de la influencia de empresarios y redes de empresarios ${ }^{11}$, en la gestión y decisión sobre política educativa (Ball, 2012, 2013; CLADE, 2015). Un tsunami neoliberal ha llegado a los distintos países de Latinoamérica y su epicentro fue Chile. Ha afectado a algunos, con distinto tipo de intensidad y en oleadas y, cual fenómeno natural, parece indiferente a la voluntad humana. Persiste, a pesar, de la oposición social y el descontento en las escuelas. Movilizaciones de estudiantes y profesores contra este tipo de reformas ha habido en Chile, Colombia, Argentina, Costa Rica, Puerto Rico, México y Brasil, sin mayor efecto en el giro de estas políticas. ¿Es posible revertir esto? 


\section{PRETEXTO: PRIVATIZACIÓN EN CHILE (¿Doctrina del shock?)}

Cómo vemos en el contexto latinoamericano la privatización pos años 90 no se ha detenido. Particularmente en Chile, continuó con la posdictadura y posterior al año 2006 pareció intensificarse. Entre el 2008 y el 2013 desaparecieron 420 escuelas municipales. El año 2005, había aproximadamente 390.000 estudiantes en la Educación Superior Privada, a 2014, (luego del Crédito con Aval del Estado) ${ }^{12}$, hay más de 800.000 estudiantes en la educación superior privada, actualmente el 70\% de la matrícula total en Educación Superior. ${ }^{13}$.

La privatización de la educación obligatoria parece irreversible. Si al año 1990 la matricula estatal alcanzaba el 54\%. Al 2014 a nivel nacional las escuelas municipales llegan a menos del 37\% de la matrícula total. En la Región Metropolitana no supera el 26\%.en promedio (Mineduc, 2014). Particularmente en comunas urbano-populares donde hay mayor concentración de población y de pobreza se ha llegado a extremos. En Puente Alto y Maipú sólo el 17,7\% de la matrícula es municipal. En La Pintana sólo un 17,9\% de la matrícula está en la educación municipal, en La Cisterna sólo 11,5\%. Alto Hospicio en el norte de Chile, sólo cuenta con sólo un 3,4\% de los/as estudiantes en establecimientos municipales, el resto está en escuelas privadas (Mineduc, 2014).

Para analizar algunas implicancias de este proceso, revisemos con mayor detalle una de estas comunas en los últimos 20 años (ver Gráfico 1)

Gráfico 1: Matrícula Educación Pública y Privada comuna La Pintana - Santiago de Chile, 1995-2014.

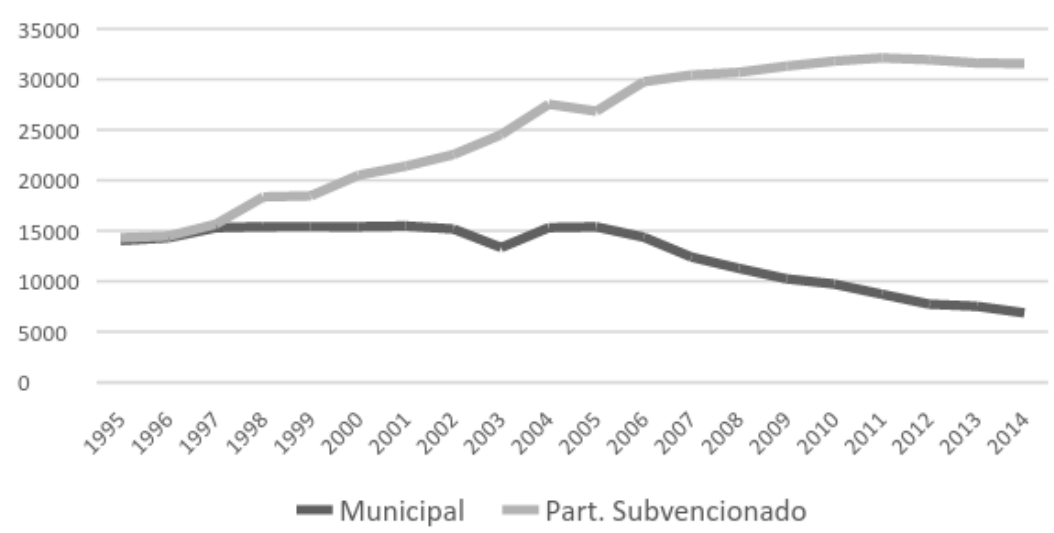

Fuente: Elaboración propia según base de datos MINEDUC (2014) 
La tendencia en los últimos 20 años, indica un crecimiento progresivo de la oferta privada, su matrícula aumentó en un 54,7\%. Situación contraria a la educación municipal, la cual ha disminuido en un 51\% a partir del año 1995 (Ver Gráfico 1). Existen 56 sostenedores a cargo de 72 establecimientos educacionales con subsidio estatal que reciben (al 2014) a una población de 38.440 estudiantes. El sistema educativo municipal administra 13 establecimientos con 6.878 estudiantes (17,9\% del total). Los 55 sostenedores privados controlan 59 establecimientos educacionales, con 31.562 estudiantes, es decir el $82,1 \%$ del total. ¿Quiénes son? ¿Seleccionan? ¿Son gratuitos? ¿Tienen fin de lucro?

Según nuestra caracterización hay cinco tipos de sostenedores privados en La Pintana (González, 2009; 2010a). Los grupos de poder ${ }^{14}$, sostenedores ligados a grupos religiosos y empresariales ${ }^{15}$, son nueve sostenedores que concentran en $26 \%$ aproximadamente de la matrícula, a través de fundaciones sin fines de lucro, seleccionan a los estudiantes y la mayoría cobra financiamiento compartido. Desde el año 1995 han comenzado a crecer en matrícula, particularmente la de menor vulnerabilidad social de la comuna, debido a su selectividad (ver Gráfico 2).

Gráfico 2. Tendencias Matrículas Poder Corporativo 1995-2014

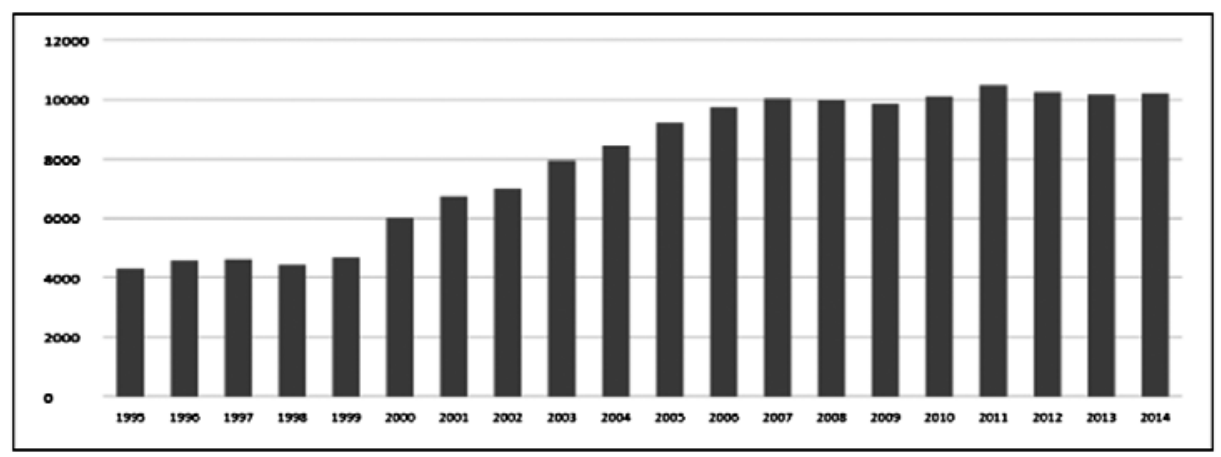

Fuente: Equipo de investigación en privatización, según bases de MINEDUC, 2014

Una característica de este grupo son los vínculos que tienen entre sí, y con otros grupos de sostenedores, empresas, grupos religioso en otras comunas del país.

Elementos que saltan a la vista son sus vínculos con los grupos de poder económico y proyectos educativos de carácter conservador y competitivos ${ }^{16}$. 
Los inversionistas son ocho sostenedores con personalidades jurídicas que declaran fines de lucro. Tienen una o más escuelas de más de 1000 estudiantes ${ }^{17}$ y sus representantes legales y /o titulares figuran en otras iniciativas comerciales y/o productivas. La mayoría de sus escuelas selecciona a sus estudiantes y cobran financiamiento compartido. Algunos son los llamados mega sostenedores ${ }^{18}$. Son empresarios, generan economías de escala, ya que varios colegios, incluso en algunos casos poseen holdings educacionales ${ }^{19}$. En el caso de La Pintana captan el 27 \% aproximadamente de la matrícula comunal, al igual que el grupo anterior, la de menor vulnerabilidad en la comuna. También se han consolidado en matrícula en estos últimos años (ver gráfico 3).

Gráfico 3: Matrícula del grupo Inversionistas (1995-2014)

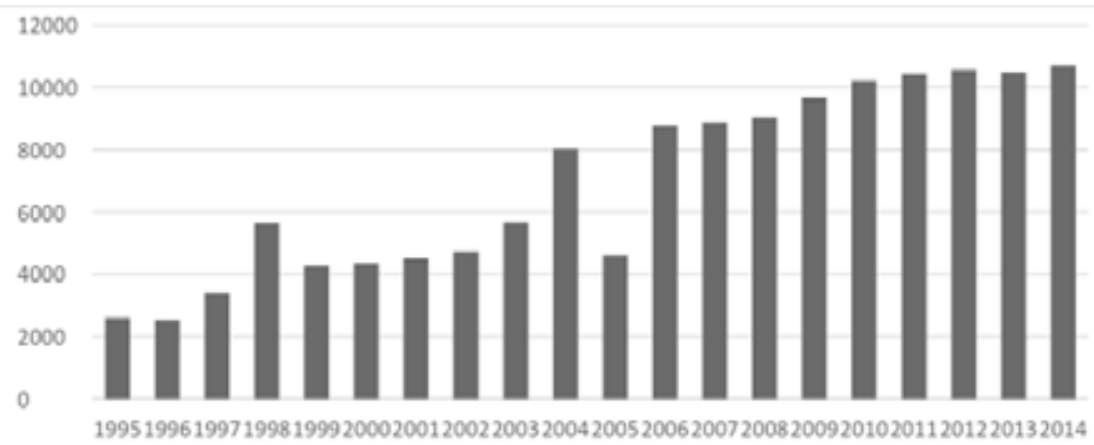

Fuente: Equipo de investigación en privatización, según base de datos MINEDUC.

El $53 \%$ de la matrícula con menor riesgo social se encuentran en las escuelas de los grupos de poder e inversionistas. Escuelas que proyectan un ejemplo de buenos resultados en sectores de pobreza y que tiene un soporte institucional (político) y económico que les da claras ventajas para mantener su gestión educativa.

Los 38 sostenedores que quedan reciben aproximadamente al $27 \%$ de la matrícula comunal, la de mayor vulnerabilidad social. El grupo mayor lo componen 24 sostenedores que tienen escuelas de lenguaje, en conjunto suman 6.000 estudiantes, es decir, al 16\% de la matrícula comunal. Lo que en sí es preocupante ya que hay un aumento explosivo, hacia el año 1998. Ese año comienza regir el aumento en las subvenciones por necesidades educativas especiales (ver gráfico 4). 
Gráfico 4: Tendencia Matrículas Escuela Especial (1995-2014), comuna de La Pintana.

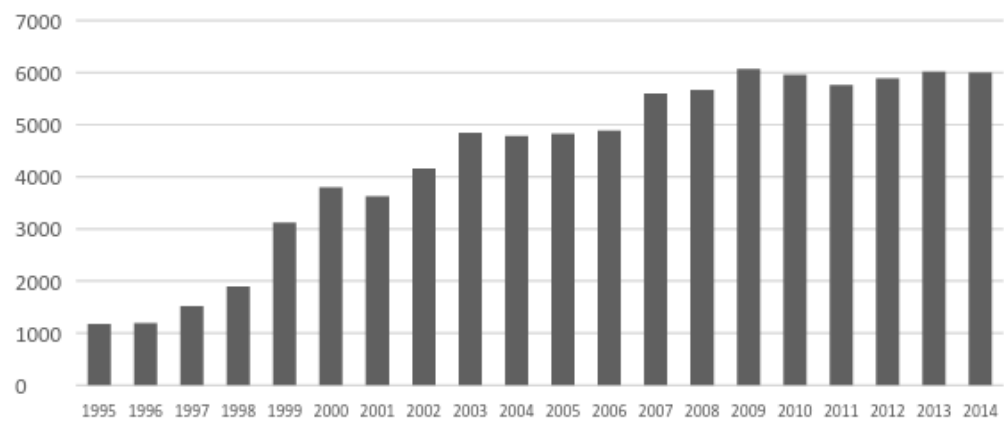

Fuente: Elaboración propia a partir de bases de datos del Ministerio de Educación (MINEDUC, 2014)

Son sostenedores pequeños que se benefician de esta subvención, sustancialmente más alta que la subvención regular, lo que les permite contar con escuelas pequeñas de menos de 250 estudiantes $^{20}$.

Hay nueve sostenedores que llamamos comerciantes, declaran fines de lucro, tienen uno o dos colegios, como máximo, de menos de 500 estudiantes. Los representantes legales y /o titulares, registran actividad comercial aparte de las escuelas, reciben en total una matrícula de 3.875 estudiantes, es decir el 10\% de la matrícula comunal. Los asalariados, tiene un solo colegio y no registran otra actividad comercial, al 2014 hay sólo 5 en esta comuna y entre todos reciben solamente a 1.888 , apenas el $5 \%$. Ninguno de ellos cobra financiamiento compartido, ni selecciona. A diferencia de los anteriortes grupos no ha ganado matrícula desde hace ya varios años, como se observa en el gráfico 3.

Gráfico 5: Tendencia Matrículas Asalariados y Comerciantes (1995-2014), comuna de La Pintana.

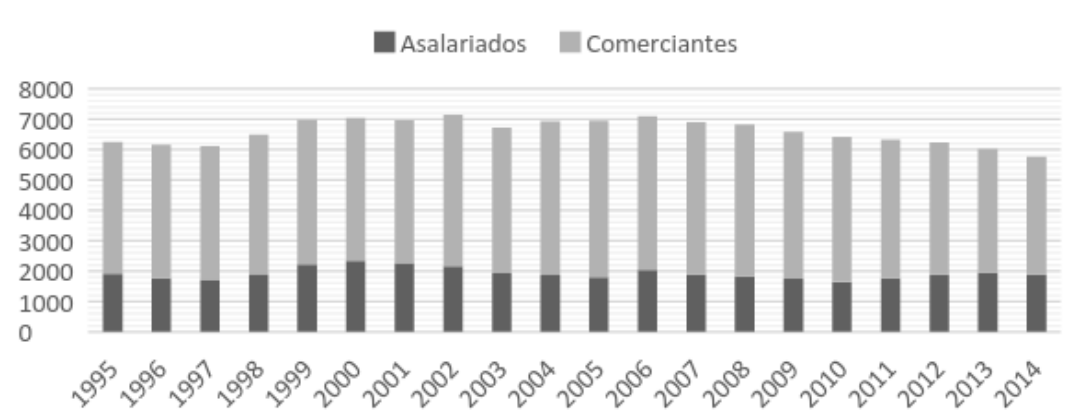




\section{LEY DE INCLUSIÓN ¿UN GIRO A LA REFORMA NEOLIBERAL?}

La Reforma Educativa Chilena es un referente para la iniciativa neoliberal en Latinoamérica. Es donde primero se aplicaron estas medidas y, por lo tanto debe ser el lugar en donde más alto desarrollo han alcanzado. Muchas de las medidas señaladas se han implementado señalando responder a la presión social, pero han terminado profundizando el modelo mercantilizador. Fue el caso del crédito con aval del estado en Chile, que pretendió dar más acceso a la educación superior y terminó por consolidar la alternativa privada. Dar un giro a este proceso en oleadas, multiforme, en construcción no debe ser algo sencillo, Monitorear los cambios requiere suspicacia, estar alertas.

Respecto a la Ley de Inclusión, el mensaje (fundamentos) del proyecto plantea como imperativo la idea de fortalecer la educación pública, sin embargo, sus tres ejes, no apuntan directamente a esto último (si entendemos la educación pública como la estatal), sino que más bien a regular algunos aspectos de la actual oferta educativa privada. ¿Esto representa un giro en el modelo?

El fin al lucro con los recursos que el Estado entrega, por medio de la subvención, a los sostenedores, la eliminación gradual y parcial de la selección escolar y la derogación gradual del sistema de financiamiento compartido, tendrían como objetivo dar un marco sustentable que permita avanzar en asegurar el derecho a una educación de calidad, reducir las desigualdades y, por sobre todo, garantizar, de manera efectiva, la libertad de los padres, madres y apoderados para elegir la educación de sus hijos.(Mensaje 131-326, 2014, p. 2).

Como vemos, el primer aspecto a destacar es que el derecho a la libertad de elección aparece como uno de los principales fundamentos y objetivos del fin a la selección y al financiamiento compartido. Al margen de lo exigido por el movimiento social, la libertad de elección encuadra el significado de la libertad de enseñanza en los marcos de la libertad de mercado. La equidad en la sociedad (en educación) se expresa en la ausencia de obstáculos a la capacidad de elegir ${ }^{21}$. Este es un primer aspecto, el ajuste a los principios de la libertad de mercado, hoy vigentes. Una reforma neoliberal de cuarta generación, en la cual la oferta privada es un derecho. Consumir es un derecho, si la educación es mercancía, consumir educación es un derecho ${ }^{22}$.

Segunda observación, en relación a la prohibición del lucro. La ley obliga a los sostenedores a convertirse en entidades sin fines de lucro, establece medidas de control sobre el gasto de la subvención y asigna un monto para 
pagar, la administración superior del establecimiento, un inédito salario para el sostenedor, para que no se vea obligado a sacar excedentes de la subvención escolar.

En consecuencia, el lucro en educación queda reducido a la ganancia que los sostenedores extraen de la subvención escolar, lo que quedaría subsanado con estas medidas, la fiscalización sobre los sostenedores por la Superintendencia de Educación y el Servicio de Impuestos Internos. No hay cuestionamiento al hecho que otros/as privados extraigan ganancias de otros fondos del Estado destinados a garantizar el derecho a la educación ${ }^{23}$. Se normaliza el lucro o la extracción de ganancias con fondos del Estado destinados a la mejora educativa $^{24}$, a la alimentación, a los textos escolares ${ }^{25}$, a la evaluación, la investigación, el acceso (como en el mencionado CAE y la financiación de los aranceles) u otros a otras áreas que puedan producir ganancias a través de la privatización. Es la legitimación de la empresa educativa, la subsunción real del sistema educativo al mercado.

Un tercer aspecto. Tras la reducción al mínimo de la oferta estatal, el sistema educativo es un campo en disputa nuevamente. La lógica de la competencia determina la provisión educativa. Quienes controlan son los que tienen mejores condiciones para competir. En el caso de La Pintana el 53 \% de la matrícula está en escuelas que pertenecen a fundaciones ligadas a los grupos de poder e inversionistas. ¿En qué los afecta a ellos los ajustes institucionales que regulan el lucro? En prácticamente nada. Muchos de ellos no lucran (Los colegios del Opus Dei lucran?), y ya tienen la solvencia institucional y financiera para afrontar los cambios ${ }^{26}$. Estos cambios afectarán a los sostenedores más pequeños (comerciantes y asalariados), que no quieran o puedan cambiar a una personalidad jurídica sin fines de lucro y que la asignación por administración sea muy baja, debido al tamaño de sus colegios. Esto podría favorecer la concentración antes señalada, mediante la cesión de escuelas $u$ otros mecanismos.

Finalmente respecto a los cambios en relación a la gratuidad (fin del financiamiento compartido o copago) y la selección de estudiantes, un gran tema son los plazos para aplicar estas medidas que pueden superar los seis $a_{n}{ }^{27}$. Por otra parte su aplicación depende de varios reglamentos que aún no son elaborados. La complejidad de los mismos radica en establecer la gradualidad y las excepciones a las medidas. Podemos esperar entonces la acción de los grupos de interés en este proceso, que sin duda será largo. 


\section{CONCLUSIÓN}

Como vemos el neoliberalismo, en términos estratégicos, es el conjunto de iniciativas varias, por el control del mercado, y por medio de él, del conjunto de ámbitos de nuestra vida, a través de la mercantilización o subsunción de la vida al capital (Negri, 2012). Este proceso en la educación se viene desarrollando hace años en oleadas de reformas que han ido transformando la institucionalidad y el sentido común educativo. En este sentido la Ley de Inclusión, parece ser una oleada más que va en esa dirección, no excluye a nadie del sistema educativo. Suma a bancos, grupos empresariales, empresas varias, en la gestión total de un sistema de provisión mixta, con un fuerte pilar privado, que se consolida bajo el control de los grupos de poder e inversionistas. Todo esto bajo la falacia de la libertad liberal, de libre empresa y libre elección. Más que un giro es un necesario ajuste, para que todo siga su curso y la escuela pública, su sentido y rol, sea solo un difuso recuerdo.

\section{NOTAS}

1. Me refiero al proceso llevado en torno al proyecto de Privatización en Chile (año 1995 al 2014), caracterización del fenómeno en tres comunas chilenas. Fondo de productividad académica FACSO. El equipo quedó conformado por Diego Parra y Rodrigo Sánchez de la Universidad de Chile y de la Cooperativa de Trabajadores Centro ALERTA e investigadores de OPECH, quienes también han colaborado en la redacción de este artículo.

2. La Ley General de Educación (2009), la Ley de Subvenciones (1998), la Ley de Jornada Escolar Completa (2004), la Ley de Subvención Escolar Preferencial (2008) y Ley de Calidad y Equidad (2011).

3. Hago la referencia a la propuesta que la Asamblea Nacional de Estudiantes Secundarios ACES del año 2011, quienes señalan en ella "(...) independiente de lo anterior y como complemento, el estado implementará un fondo de recuperación de la educación para la educación pública. Se trata de una inversión que se realiza una sola vez, para recuperarse del estado de devastación del terremoto neoliberal" (p.5)

4. Lo decía el ministro el día de su aprobación. "Las reformas estructurales siempre son extremadamente complejas", sin embargo, enfatizó que "las virtudes del modelo que estamos estableciendo para el conjunto de la educación, están fundadas de manera contundente en toda la literatura y en la experiencia empírica de todos los países que han logrado construir buenos sistemas educacionales". (Ver Senado aprueba 
Reforma en: http://www.cpeip.cl/index2.php?id_seccion=10\&id_portal=1\&id_ contenido=30597)

5. En Chile, con la constitución del año 80 se cambia el rol garante del Estado a uno subsidiario. Además se instala proteger la libertad de enseñanza, como el derecho privado a abrir y gestionar establecimientos educacionales, con garantía constitucional, no así el derecho a la educción (art.19 a $21^{\circ}$ ).

6. El año 1972 el estado en educación gastaba el equivalente al 7\% del PIB, con una cobertura mucho menor a la actual. En 1980 la inversión se había reducido al 4,6\% del PIB y el año 1990, solo llego 2,9\% en pleno proceso de expansión de la matrícula. Actualmente el Estado gasta aproximadamente el 4 \% de su PIB en Educación, teniendo una cobertura total en enseñanza básica y media.

7. Sobre la evolución de las políticas educativas neoliberales en los sistemas educativos latinoamericanos ver: Las Reformas Educativas en los países del CONOSUR Un Balance Critico. Buenos Aires: CLACSO, abril 2005. Una interesante mirada desde las organizaciones sociales de docentes de Brasil, Uruguay, Argentina y Chile. Otro material relevante es el trabajo los investigadores ligados CLACSO, que desde hace año han dado cuenta de la evolución de las políticas, llamadas neoliberales en los sistemas educativos Latinoamericanos desde los año 90 en adelante. Interesante síntesis analítica se encuentra en Gentili et.al. (2009). Políticas de privatización, espacio público y educación en América Latina. Rosario: Homo Sapiens Ediciones.

8. Con esta frase se hace referencia a la utilización marxista del término subsunción que hace Marx en El Capital (tomo I) al describir el proceso por el cual el trabajo se transforma en herramienta de valorización capitalista y abandona otras funciones. Toni Negri retoma la noción y señala la actual subsunción real de la vida al capital, esto es la subordinación de todos los aspectos de la vida humana a la reproducción capitalista. Ver Negri, T. (2012). Biocapitalismo. Entre Spinoza y la Constitución Política del Presente. Buenos Aires: Quadrata.

9 La Nueva Gestión Pública (NGP) constituye una tendencia que incorpora aspectos propios de la lógica de los negocios privados en la administración y recompensas para orientar a las unidades o empleados al logro de metas pre-establecidas (CLADE, 2015, p. 20).

10. La orientación de la escuela a resultados medibles, la introducción del mecanismo de evaluación, management y gerenciamiento educativo (Grinberg, 2008). En síntesis, la llamada despedagogización de la escuela (Mejía, 2008). 
11. Se consolida en Latinoamérica la incidencia de los grupos empresariales en el control de las políticas educativas, incluso a nivel transnacional. Es el caso de la red "REDUCA", presente en 13 países latinoamericanos, entre ellos Chile, donde está su secretaría ejecutiva (Fundación 2020). REDUCA reúne a grupos empresariales o agrupaciones financiadas por grupos empresariales, que se organizan para incidir en la agenda educativa de sus respectivos países. Ver http://www.reducaal.net/ y entrevista a Erika Moreira "Se amplía red vinculada con empresas para incidir en políticas de educación” http://www.jornada.unam.mx/2012/04/02/ sociedad/040n2soc

12. Crédito con Aval del Estado CAE: Medida propuesta por el gobierno de Ricardo Lagos el año 2005 que permite a los/as estudiantes optar por crédito bancario para pagar los aranceles de la educación superior, en donde cada estudiantes podía elegir qué universidad estudiar, cuyo aval era el propio Estado. Mas sobre las consecuencias de esta medida ver OPECH (2012) La Reforma al CAE: Se CAE la Educación Superior Pública http://www.centroalerta.cl/la-reforma-al-cae-se-cae-la-educacion-superiorpublica-2/

13. En Chile las Universidades estatales en general son más selectivas y no han ampliado significativamente sus cupos. En consecuencia las Instituciones privadas no tradicionales han desplazado a las instituciones del Consejo de Rectores como las instituciones de mayor tamaño. En 2005, cuatro universidades del Consejo de Rectores eran parte de las 10 instituciones con mayor matrícula (Universidad de Chile, Universidad de Santiago, Universidad de Concepción y Pontificia Universidad Católica). En 2011, sólo la Universidad de Chile ocupa un lugar entre las 10 instituciones de mayor matrícula, el resto son privadas

14. Los grupos de poder son fundaciones vinculadas a grupos empresariales financieros e industriales y/o a grupos religiosos como la Sociedad de Instrucción Primaria vinculada al grupo Matte, o Belén Educa, vinculada al arzobispado de Santiago.

15. Por ejemplo del sector inmobiliario, forestal, financiero, industrial, que forman parte del directorio de estas fundaciones o son donantes y/o financistas. Algunas de estas fundaciones reciben recursos por la ley 19.247 de donaciones con fines educativos, fondos que tienden a concentrase en este tipo de escuelas. Por ejemplo, en el bienio 2006-07 el fondo tuvo casi 30.000 dólares en la Región Metropolitana, el 90\% se lo repartieron entre 55 escuelas particulares subvencionadas el resto fue a la educación municipal. Así, año tras año este tipo de fondos se concentran en las mismas escuelas. (Datos Intendencia Metropolitana de Santiago- Chile).

16. Mas sobre este tema en González J. y otros (2012) El proyecto educativo del complejo religioso empresarial y las políticas de la Nueva Derecha. Revista de 
Pedagogía Militante Diatriba $N^{\circ} 2$, Editorial Quimantú, Agosto, pp. 22-35. En http:// colectivodiatriba.files.wordpress.com/2012/12/revista-diatriba-nc2ba21.pdf

17. La razón de esto nos la relató un sostenedor de este grupo en entrevista con el equipo de investigación "El negocio es tener colegios de más de 1000 estudiantes, si no, no es negocio. Nosotros apostamos a obtener una ganancia anual entre un 15\% a 20\%, no menos" (Entrevista a sostenedor miembro de CONACEP, año 2009).

18. Por ejemplo aparece en nuestro estudio la Sociedad Educacional e Investigaciones Pedagógicas Galvarino Ltda., ligada al grupo Cifuentes Narváez, parte de los llamados megasostenedores, este grupo que parte el año 1974 con Filomena Narváez, una de las fundadoras de CONACEP, hoy, en manos de sus hijos, es un holding educacional, que tras 10 Sociedades distintas sostiene 19 establecimientos en otras comunas, además sostiene una Universidad Privada y son propietarios de distintas sociedades inmobiliarias, agropecuarias y comerciales. Otro ejemplo es Luis Huneeus que sostiene el Colegio Jorge Huneeus Zegers. Tiene sólo un establecimiento pero de más de 3400 estudiantes, es el más grande en la comuna. El sostenedor es un político local y acaudalado empresario, militante del partido de derecha, Renovación Nacional, que actualmente es concejal de la comuna, vinculado a distintas actividades comerciales y empresariales.

19. Más sobre esto ver reportaje Javier Rebolledo, Megasostenedores con Promedio Rojo. Los millonarios de la educación en http://www.opech.cl/editoriales/2006_11/ megasostenedores.pdf. Un reportaje actual ver CIPER "Así operan los siete grupos de megasostenedores” en: http://www.rebelion.org/noticia.php?id=185926

20. La subvención escolar dada por niños diagnosticados con necesidades educativas especiales registradas en el decreto 170, al menos duplica la subvención regular. Esta problemática de claro diagnóstico y la implicancia que puede tener la normativa, en tanto incentivo perverso, ya está siendo investigada y denunciada en los medios académicos. Las consecuencias de esto en esta comuna son evidentes. Las implicancias para la población deben ser comentadas en más detalle de crecimiento, sin embargo esto excede el objetivo de este trabajo y será retomado en análisis posteriores.

21. Señala el proyecto en sus fundamentos "Este sistema restringe gravemente la libertad de enseñanza; los padres, madres y apoderados no escogen establecimientos conforme al proyecto educativo que sea de su preferencia, sino que sólo pueden optar entre aquellos que puedan pagar. El ejercicio de esta libertad está condicionado por los ingresos de las familias y restringido por la estructura diferenciada de copago y la inequidad en la distribución espacial de las escuelas. Por ello, es que este proyecto debe garantizar la libertad de enseñanza y hacerla efectiva, a fin que todos los padres, madres y apoderados del país, independientemente de sus ingresos, puedan elegir 
los establecimientos educacionales que sean de su preferencia” (Mensaje 131-326, p.13, 2014). Similar argumento se utiliza respecto a la eliminación de la selección y en relación al contenido de la libertad de enseñanza en varios puntos del mensaje y la ley.

22. El 2014 la Confederación de Padres y Apoderados CONFEPA, organización animada por la derecha convoca una masiva marcha por la libertad de elegir .Movilizando recursos logran sacar a más de 30.000personas a la calle. Erika Muñoz, presidenta de la Confepa, señaló a Radio Futuro que "nosotros estamos de acuerdo con que nuestros hijos puedan seguir con subvención y a que nuestros hijos puedan tener el proyecto educativo que queramos elegir"

23. Curiosamente en los fundamentos de esta ley, el problema con el lucro es la ausencia de evidencia sobre buenos resultados en establecimientos con fines de lucro a nivel nacional e internacional y la probable merma en la calidad de la enseñanza que implica destinar los fondos de las escuelas en otros fines (ganancias). “(...) la evidencia reciente muestra que los establecimientos particulares subvencionados con fines de lucro tienen en general peores resultados que los establecimientos sin dicho fin, o bien, muestran un efecto neutro o muy mínimo (sobre resultados escolares medidos en los test SIMCE). (......) "A nivel internacional, la evidencia muestra que son escasos los ejemplos de países que permiten que los sostenedores de los establecimientos educacionales puedan utilizar, para sus intereses particulares, recursos destinados a mejorar los procesos y condiciones de aprendizaje. Incluso en los casos de sistemas similares al chileno (donde priman mecanismos de mercado para orientar los procesos educativos), y, salvo muy pocas excepciones, los establecimientos privados deben ser sin fines de lucro". (Mensaje, 131-326, p. 20, 2014).

24. Es importante señalar que durante la tramitación de la ley se hizo la indicación de que estas agencias fueran entidades sin fines de lucro, lo que finalmente fue desestimado.

25. Cabe aquí destacar el caso de los textos escolares en donde se ha ido constituyendo un oligopolio durante estos últimos 15 años. Al 2015 tres empresas controlan de los fondos estatales destinados a este ítem. Santillana con el 32\%, Cal y Canto con el 12\% y Zig-Zag con el 78,8\% (datos entregados por ley transparencia equipo investigación, MINEDUC 2015)..

26. La ley asignará además un monto para la administración superior del establecimiento. La cual deberá tener un valor en relación a la complejidad de la función, cantidad y tamaño de los establecimientos y el valor de mercado para ese tipo defunciones.

27. Como ya señaló Andrés Palma encargado de la reforma en los medios de comunicación el 9 de Agosto del 2015 "La gradualidad de esta ley es gigantesca. Ningún cambio 
es obligatorio en 2016. Pasar a gratuidad no tiene fecha. Hay colegios que pueden estar 20 años más con copago. Para pasar a ser sin fines de lucro, quedan dos años y medio de plazo. Para ser propietario de los inmuebles, hay entre 3 y 6 años adicionales, y durante todo ese período pueden acceder a créditos con la garantía Corfo. No hay ningún plazo apurado" Ver en http://www.economiaynegocios.cl/ noticias/noticias.asp?id=171252. En relación a la selección, los plazos son también extensos, hasta 6 años, y desde las comunas más pequeñas, a las más grandes, donde es más grave el problema de la segregación.

\section{REFERENCIAS BIBLIOGRÁFICAS}

Assaél, J., R. Cornejo, J.

La empresa educativa Chilena. Educación

González, J. Redondo, R. y Sociedad. [online]. 2011, vol.32, n.115, Sanchez, y M.Sobarzo., 2011 pp. 305-322.

Ball, S. y Corbalán, F., 2013

Un nuevo escenario para la política y el negocio educativos. Cuadernos de Pedagogía, n. 435, p. 1-5, jun. 2013.

Ball, S, 2012.

Global Education Inc. New Policy Networks and the neoliberal imaginary.

New York: Routledge

Base datos MINEDUC, 2014

Base de datos y fuentes

Investigación, 2014

Observatorio de Políticas

Educativas, 0. L., 2005

CLADE, 2015
Privatización de la Educación en Chile el caso de 2 comunas. Fondo de productividad académica FACSO.

Las reformas educativas en los países del Cono Sur. Informes Nacionales. Buenos Aires. CLACSO

Mapeo sobre tendencias de la privatización de la educación en América Latina y el Caribe. Campaña Latinoamericana por el Derecho a la Educación CLADE, Sao Paulo, 2015. MIMEO 
Corbalán, F., J. González, y S. Identificación de la Propiedad y Ligüeño, 2009 Dinámica de la Oferta Educativa Particular Subvencionada de la Región Metropolitana. Revista del Magíster en Análisis Sistémico Aplicado a la Sociedad No 20. Mayo. Facultad de Ciencias Sociales Universidad de Chile. En: http://www.revistamad.uchile.cl/20/ corbalan_05.pdf

Foro por el Derecho a la Educación, 2015

Gentili et.als. 2009

González, J. et als. 2010a

González, J. 2010b

González J. y R. Sánchez, 2011

González J. et.als. 2012
Análisis Crítico Ley de Inclusión.

Políticas de privatización, espacio público y educación en América Latina. Rosario: Homo Sapiens Ediciones.

Gubernamentalidad y provisión educativa privada en chile. Reflexiones a partir de la identificación de la propiedad y dinámica de la oferta educativa privada en la Región Metropolitana En Sujetos y actores Sociales: Reflexiones en el Chile de Hoy». Kemy Oyarzun Compiladora Subprograma Domeyko Sujetos y Actores Sociales. Universidad de Chile. Año

La educación en el liberalismo autoritario, la mercantilización como estrategia de control. En Cassigoli, I. y Sobarzo, M. eds. Biopoliticas del Sur. Editorial ARCIS, pp. 251- 260.

2011: Aportes para interpretar una década de lucha por autoeducación. Editorial Quimantú. Santiago Septiembre, 2012 P. 180185.

El proyecto educativo del complejo religioso empresarial y las políticas de la Nueva Derecha. Revista de Pedagogía Militante. Diatriba №2, Editorial Quimantú, Agosto, pp. 22-35. 
González J., M. Reyes, R.

Sánchez, M. Sobarzo 2014

Grimberg, S. 2008

Mejía, M. 2008

Mensaje, 2014

Negri, T. 2012

OCDE 2004

OPECH 2009 2006
La inanición electoral chilena y la nueva forma de hacer política del movimiento social. Revista Contrapunto $\mathrm{N}^{\circ} 4$, Universidad de La República Uruguay. En http://www.contrapunto.edu.uy/ images/pdf/contrapuntos/Original_ contrapunto_4_mayo_final.pdf

Educación y Poder en el siglo XXI. Gubernamentalidad y pedagogía en las sociedades de gerenciamiento. Buenos Aires: Ed. Miño y Dávila

Guerra, S. L., \& Chávez, M. F. Las reformas educativas neoliberales en Latinoamérica. Revista electrónica de investigación educativa, 81.

El sistema de subvenciones en educación: la experiencia chilena Vol. 99. Santiago de Chile: Centro de Estudios Públicos.

Las Pedagogías Críticas en Tiempos de Capitalismo Cognitivo. Cartografiando las resistencias en educación. http:// www.cepalforja.org/sistem/documentos/ pedagogias_criticas.pdf

Mensaje presidencial $N^{\circ} 131-362$

Biocapitalismo. Entre Spinoza y la Constitución Política del Presente. Buenos Aires: Quadrata.

Revisión de Políticas Nacionales de Educación: Chile. Paris: OCDE.

Las Luchas del Movimiento por la Educación en Chile y la reacción neoliberal. En Chile, La vitrina del neoliberalismo: Otro Chile es urgente, posible y necesario. Brill, Critical Global Studies Series Ximena de la Barra, Editor 2010. Pp. 153 - 178. 
Pagano, A., I. Sverdilk, , P.

Costas, 2007

Puiggrés, 2014

Riesco, 2011

Sader, E., 2006
Participación e incidencia de la Sociedad Civil en las Politicas Educativas: El caso Argentino. 1ra Edición, Libros FLAPE.

Educación y sociedad en América Latina de fin de siglo: del liberalismo al neoliberalismo pedagógico. Estudios Interdisciplinarios de América Latina y el Caribe.

La necesidad de reconstruir el sistema nacional de educación público desmantelado por la dictadura y la LOCE. Pensamiento Educativo, nº40, pp. 243-261

Conferencia inaugural encrucijadas de la educación Latinoamericana. Foro Latinoamericano de Políticas Educativas. Santiago de Chile 
